\title{
Memoria neuromuscular y control motor en odontología
}

Beltrán-Neira RJ. Memoria neuromuscular y control motor en odontología. Rev Estomatol Herediana. 2011; 21(4):240.

Roberto J. Beltrán Neira

Profesor emérito, Profesor extraordinario investigador. Facultad de Estomatología. Universidad Peruana Cayetano Heredia.
Memoria neuromuscular, memoria muscular, control motor o aprendizaje psicomotor son expresiones usadas indistintamente en la literatura.

El conocimiento de cómo se adquiere y almacena el saber psicomotor es útil para mejorar la enseñanzaaprendizaje-evaluación de los procedimientos clínicos odontológicos. La tradición en la adquisición de destrezas psicomotoras se basa en la observación de un modelo dinámico y su repetición hasta alcanzar un dominio automatizado del procedimiento aprendido.

Como profesores nos interesa explorar distintas formas para alcanzar el mejor resultado en el menor tiempo. Una persona puede intentar ejecutar una actividad a partir de su descripción oral o escrita, sin embargo la experiencia enseña que el olvido y la interpretación del lenguaje constituyen barreras importantes. La dificultad está en transformar un conocimiento enunciado en un procedimiento psicomotor. Puede ser que el aprendiz alcance a reproducir los movimientos descritos oralmente o por escrito, pero seguramente le tomará un tiempo excesivo y carecerá de la crítica de un observador experimentado que lo ayude a interpretar lo escuchado del profesor o leído en un texto.

Mejor opción es disponer que el aprendiz observe la ejecución del procedimiento por un práctico diestro. La observación puede ser repetida o puede ser complementada con demostraciones grabadas, las cuales tienen la ventaja de que acercan más a la escena en demostración. Las grabaciones ofrecen también la facilidad de ser vistas repetidamente.

Una vez observado el procedimiento, toca al aprendiz ensayar una primera ejecución en las condiciones más parecidas a las habidas en la demostración. (Esta primera ejecución puede ser en material inerte o en paciente) La primera ejecución tiene que ser observada de cerca por un instructor. La repetición del procedimiento bajo condiciones ordinarias logrará que el cerebro almacene en su repertorio la destreza aprendida como memoria neuromuscular.

Los avances de las neurociencias han mejorado la comprensión de en qué consiste la memoria neuromuscular. Sabemos que la memoria está dispuesta como sistema múltiple en el cerebro, de modo que la comunicación interneuronal permite construir una réplica del procedimiento aprendido. Si bien el cerebelo constituye el elemento protagónico, son las redes formadas por la comunicación entre las neuronas sensitivas y motoras las que permiten reproducir la destreza aprendida. Es reconocido que las destrezas psicomotoras aprendidas tienen una gran resistencia al olvido. Por ejemplo, la persona que aprende a manejar una bicicleta o un automóvil, podrá volver a hacerlo a pesar de que haya transcurrido mucho tiempo desde la última vez que lo hizo. En clínica odontológica la destreza aprendida se mantiene sin mayor deterioro a pesar de que haya habido un lapso notable entre ejecuciones, puede haber una disminución en la velocidad del procedimiento pero la tarea se cumple a cabalidad.

La institución educativa odontológica tiene la responsabilidad de emplear los mejores modelos para el aprendizaje de los procedimientos, de modo que lo aprendido no incluya errores de movimiento que perjudiquen la calidad del producto, molesten indebidamente al paciente o extiendan innecesariamente el tiempo de ejecución. Esta responsabilidad se traduce en el cumplimiento riguroso de los protocolos de procedimientos psicomotores, lo cual queda a cargo de los instructores clínicos. Sin embargo, la existencia de protocolos bien establecidos y probados, no es obstáculo para que se siga investigando, a fin de perfeccionar los modelos de aprendizaje. Los instructores clínicos tienen en este campo grandes oportunidades para investigar y aportar al bagaje educativo de su institución en procura de excelencia.

Los módulos de aprendizaje clínico intensivo creados y experimentados durante cuarenta años en la Facultad de Estomatología de la Universidad Peruana Cayetano Heredia son el resultado de la información obtenida, cuestionamiento, intuición, experimentación y evaluación. Cada módulo se dedica al aprendizaje de una función odontológica, incluyendo aspectos cognitivos, psicomotores, afectivos y volitivos.

Queda claro que la actuación psicomotora tiene una base científica que respalda la actividad, de ahí que cada módulo de aprendizaje clínico intensivo comience con la revisión y aplicación del conocimiento a fin de que el procedimiento psicomotor quede sustentado en todas sus facetas. 